\title{
Optimization of an Integrated Wavelength Monitor Device
}

\author{
Pengfei Wang ${ }^{1,2 *}$, Gilberto Brambilla ${ }^{1}$, Yuliya Semenova ${ }^{2}$, Qiang $\mathrm{Wu}^{2}$, Gerald Farrell ${ }^{2}$ \\ ${ }^{1}$ Optoelectronics Research Centre, University of Southampton, Southampton SO17 1BJ, United \\ Kingdom \\ ${ }^{2}$ Photonic Research Centre, Dublin Institute of Technology, Kevin Street, Dublin 8, Ireland
}

\begin{abstract}
In this paper an edge filter based on multimode interference in an integrated waveguide is optimized for a wavelength monitoring application. This can also be used as a demodulation element in a fibre Bragg grating sensing system. A global optimization algorithm is presented for the optimum design of the multimode interference device, including a range of parameters of the multimode waveguide, such as length, width and position of the input and output waveguides. The designed structure demonstrates the desired spectral response for wavelength measurements. Fabrication tolerance is also analysed numerically for this structure.
\end{abstract}

Keywords: Integrated waveguide, multimode interference, edge filter, wavelength monitor

\section{INTRODUCTION}

Various techniques for wavelength measurement have been recently proposed for deployment in Dense Wavelength Division Multiplexing (DWDM) for optical communication systems and for interrogation of optical sensing systems based on a wavelength-shift mechanism, such as fiber Bragg grating (FBG) sensing systems. Integrated wavelength monitors based on planar lightwave circuits have a range of advantages, such as low fabrication cost, compact size, high scalability, fast response, and also physical robustness in comparison with wavelength measurement systems based on bulk components. To date, the integrated wavelength monitors reported in the literature include directional couplers, multimode interference (MMI) couplers, arrayed waveguide gratings and a Y-branch with an Sbend arm [1-4].

An MMI coupler has a simple structure and is conventionally used as a beam splitter/combiner. An integrated wavelength monitor based on an MMI structure exploits the wavelength dependence of interference occurring within the multimode waveguide section. Although the well-known self-imaging principle is useful in designing the MMI structure as a splitter/combiner [5], but it is beyond the capability of this method to design an MMI structure to act as an edge filter for wavelength measurements. A design procedure [6] has been reported previously where a suitable length for the multimode waveguide section can be found through scanning the multimode waveguide length to achieve the optimal MMI parameters at a given waveguide width.

In this paper we present an alternative design method for an MMI structure based wavelength monitor that includes the following steps: 1) generation of a new set of parameters; 2) acceptance of the new parameters; 3) cooling schedule and termination. In the optimization of the MMI structure, both the length and width of the multimode waveguide section and positions of the input and output waveguides are considered, and a global optimization algorithm associated with a simulated annealing algorithm [7-10] is employed. The fabrication tolerance of the designed structure is also analysed in this paper.

* pw3y09@orc.soton.ac.uk; phone: +44 238059 3954; fax: +44 2380593149 


\section{OPTIMIZATION OF A MULTIMODE INTERFERENCE STRUCTURE FOR WAVELENGTH MEASUREMENT}

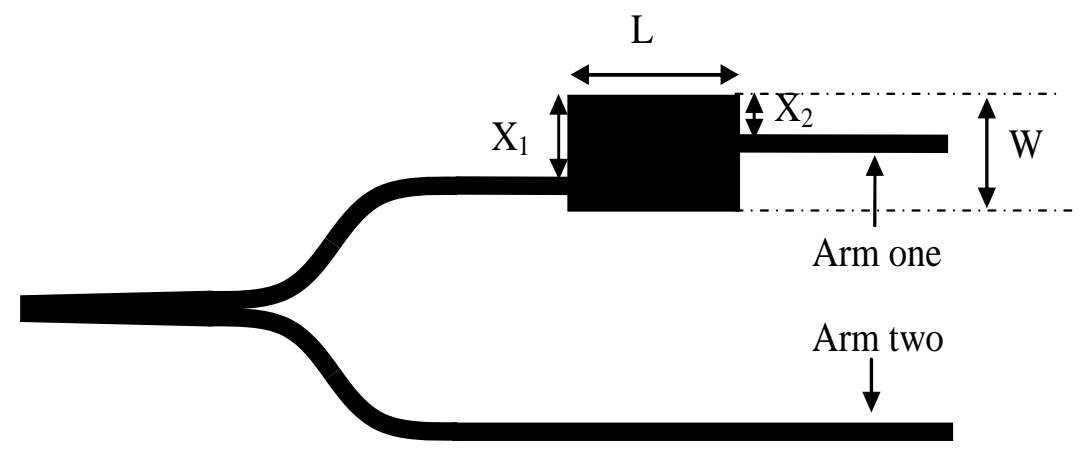

(a)


(b)

Fig.1. a) Schematic configuration of a ratiometric wavelength monitor with an MMI structure in one arm; b) desired spectral response of the two arms and output ratio between the two arms.

As shown in Fig. 1, the integrated wavelength monitor is a Y-branch with one arm serving as a reference and the other arm contains an MMI structure acting as an edge filter. The corresponding physical parameters are as follows: the waveguide cross-section is $5 \mu \mathrm{m} \times 5 \mu \mathrm{m}$. The considered wavelength range is from $1500 \mathrm{~nm}$ to $1600 \mathrm{~nm}$ and the desired spectral response of the edge filter is from 5 to $20 \mathrm{~dB}$. The search range for the width of the MMI section is from 20 to $50 \mu \mathrm{m}$. As shown in Fig.1, a linear and monotonically incremental spectral response for the ratio over the wavelength range is desirable, i.e.,

$$
L_{s}(\lambda)=L_{s 1}+\left(\frac{\lambda-\lambda_{1}}{\lambda_{2}-\lambda_{1}}\right)\left(L_{s 2}-L_{s 1}\right)
$$

where $L_{s 1}$ and $L_{s 2}$ is the transmission loss at the wavelength $\lambda_{1}$ and $\lambda_{2}$, respectively. To optimise the multimode waveguide structure for the wavelength measurement application, the length and width of the multimode section, the 
positions of the input and output waveguides, i.e., $\left(L, W, x_{1}, x_{2}\right)$ presented in Fig.1a, are considered in the optimization procedure and the objective function is defined as:

$$
F_{o b j}=\int_{\lambda_{1}}^{\lambda_{2}}\left|\bar{L}_{s}\left(\lambda, W, L, x_{1}, x_{2}\right)-L_{s}(\lambda)\right| d \lambda
$$

where $L_{s}(\lambda)$ is the desired transmission as presented by Eq.(1) and $\bar{L}_{s}\left(\lambda, W, L, x_{1}, x_{2}\right)$ is the actual transmission of the multimode waveguide structure. The transmission response of the multimode interference structure is calculated with the beam propagation method. The beam propagation method is chosen as it takes account of the leaking radiation occurring at the interface between the multimode and singlemode waveguides in the MMI section as opposed to the guided-propagation analysis $[5,11]$ which does not consider this radiation.

Reasonable searching ranges of these parameters have to be defined before implementing the optimization program. Although these four parameters are independent from each other, the sensible searching ranges of the parameters $L, x_{1}$ and $x_{2}$ are related to the width $W$ of the multimode waveguide section. For example, for a given width $W$ of the multimode section, the input and output waveguides should be located within $\left[\begin{array}{ll}0 & W\end{array}\right]$, which means that the searching ranges for these two parameters depend on the width of the MMI section. Furthermore from the selfimaging principle, it can be seen that the beat length $L_{\pi}$ between the fundamental and first-order eigenmodes of the MMI section has a close relationship with the width parameter $W\left(L_{\pi} \approx \frac{4 n W^{2}}{3 \lambda}, n\right.$ is the refractive index of waveguide core and $\lambda$ is the wavelength in free-space), which also suggests that the searching range of the length parameter depends on the width of the MMI section. Therefore, for convenience when implementing the optimization, a new set of variables are defined as $A=\left\{A_{i}, i=1,2,3,4\right\}$ with a searching range from $A^{L}$ to $A^{U}$ based on the structural parameters $\left(L, W, x_{1}, x_{2}\right)$. The corresponding relationships between these two sets of variables are: 1) $A_{1}=W, A_{1}^{L}$ and $A_{1}^{U}$ are determined by the waveguide parameters in specific optimization examples; $A_{2}=L / L_{\pi}$ and the corresponding searching range is chosen around the beat length, e.g. $A_{2}^{L}=0.5$ and $A_{2}^{U}=1.5$ (using multiple beat lengths is also possible but it will increase the length of the device); 3) $A_{3}=x_{1} / W$, $\left.A_{3}^{L}=0, A_{3}^{U}=0.5 ; 4\right) A_{3}=x_{2} / W$ and the searching range is $A_{4}^{L}=0, A_{4}^{U}=1.0$ (note: the searching ranges for variables $A_{3}$ and $A_{4}$ are interchangeable).

The simulated annealing method is chosen as the optimization method in this paper. It is a stochastic searching technique which can work without being trapped by a local minimum. There are three key procedures in implementing the simulated annealing optimization:

1) Generation of a new set of parameters. A new parameter $A_{i}^{\text {new }}$ is generated from the old parameter $A_{i}^{\text {old }}$ according to the formula $A_{i}^{\text {new }}=A_{i}^{\text {old }}+y_{i}\left(A_{i}^{U}-A_{i}^{L}\right)$, where $y_{i}$ is determined by $y_{i}=\operatorname{sgn}(u i-0.5) T(k)\left(\left(1+\frac{1}{T(k)}\right)^{\left|2 u_{i}-1\right|}-1\right)$, where $u_{i}$ is a random number uniformly distributed in the range $[0,1], T(k)$ is the annealing temperature at time $k$. 
2) Acceptance of the new parameter. Compare the value of the objective function $F_{o b j}\left(A^{\text {new }}\right)$ with that of $F_{o b j}\left(A^{\text {old }}\right)$. If $F_{o b j}\left(A^{\text {new }}\right)<F_{o b j}\left(A^{\text {old }}\right)$, the generated set of variables are accepted. If $F_{o b j}\left(A^{\text {new }}\right) \geq F_{o b j}\left(A^{\text {old }}\right)$, we compare $\exp \left[-\left(F_{o b j}\left(A^{\text {new }}\right)-F_{o b j}\left(A^{\text {old }}\right)\right) / T(k)\right]$ with $r(r$ is a random number uniformly distributed in range [0, 1]). If $\exp \left[-\left(F_{o b j}\left(A^{\text {new }}\right)-F_{o b j}\left(A^{\text {old }}\right)\right) / T(k)\right] \geq r$, we also accept the newly generated parameters; otherwise, $A^{\text {new }}$ is rejected.

3) Cooling schedule and termination. After the above two steps, i.e., parameter generation and acceptance process, the temperature is cooled down with the exponential annealing schedule, $T=T_{0} \exp \left(-c k^{1 / 4}\right)$, where $c=m \exp (-n / 4)$, and the final temperature is defined as $T_{f}=\exp (-m)$ to terminate the optimization program. The cooling down schedule of the simulated annealing optimization can be adjusted through tuning the $m$ and $n$ parameters.

\section{NUMERICAL EXAMPLE AND FABRICATION TOLERANCE ANALYSIS}

As a numerical example for the above integrated wavelength monitor, a buried silica-on-silicon waveguide is chosen and the corresponding parameters are: refractive indices for the waveguide core and cladding are $n_{\text {core }}=1.454$ and $n_{\text {clad }}=1.445$. The singlemode waveguide cross-section is $5 \mu \mathrm{m} \times 5 \mu \mathrm{m}$. The considered wavelength range is from $1500 \mathrm{~nm}$ to $1600 \mathrm{~nm}$ and the desired spectral response of the edge filter is from 5 to $20 \mathrm{~dB}$. The searching range for the width of the MMI section is from 20 to $50 \mu \mathrm{m}$. Based on the above proposed annealing optimization program, the final results for the multimode waveguide section are $\mathrm{W}=46 \mu \mathrm{m}, \mathrm{L}=4402 \mu \mathrm{m}, \mathrm{x} 1=20.4 \mu \mathrm{m}$, and $\mathrm{x} 2=40.7 \mu \mathrm{m}$. With this set of structure parameters, the corresponding spectral response is plotted in Fig. 2 using a solid line with a transmission from 7.17 to $21.3 \mathrm{~dB}$ over the wavelength range from 1500 to $1600 \mathrm{~nm}$. For comparison the desired spectral response of the MMI structure is presented in Fig. 2 by way of a dashed line. From Fig. 2 it can be seen that the designed structure has a suitable spectral response for wavelength measurement. For this configuration, the theoretical reference arm has a constant transmission of $3 \mathrm{~dB}$. Therefore, the ideal ratio curve is the transmission of the above MMI structure minus 3 dB.

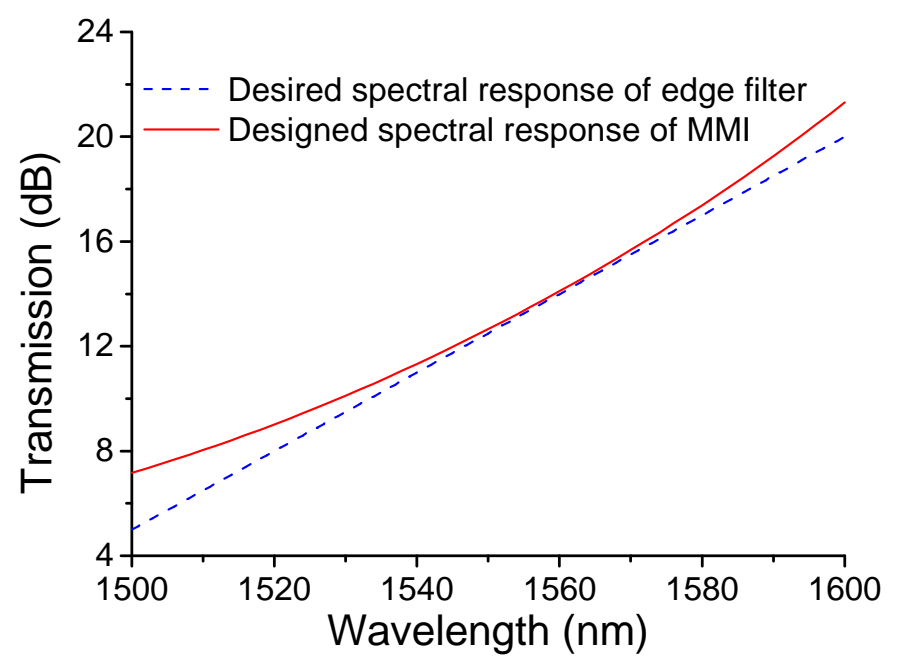

Fig. 2. Spectral response of the optimised MMI structure. 
To illustrate the light propagation within the whole device, corresponding simulation results at wavelengths of 1500 and $1600 \mathrm{~nm}$ obtained with the beam propagation method are presented in Fig.3a and Fig.3b respectively. It can be seen that the transmission responses of the output arm with the MMI section are different at these two wavelengths. The designed MMI filter results in a transmission loss of $7.17 \mathrm{~dB}$ at a wavelength of $1500 \mathrm{~nm}$ (Fig.3a) and at a wavelength of $1600 \mathrm{~nm}$ the corresponding transmission loss is $21.3 \mathrm{~dB}$ (Fig. $3 \mathrm{~b}$ ).

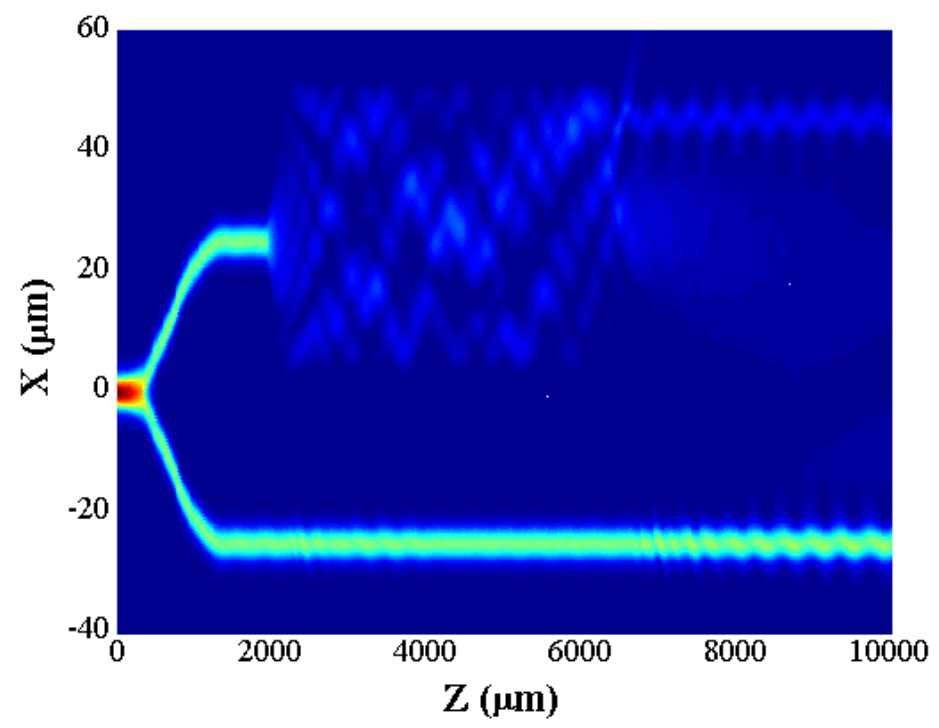

(a)

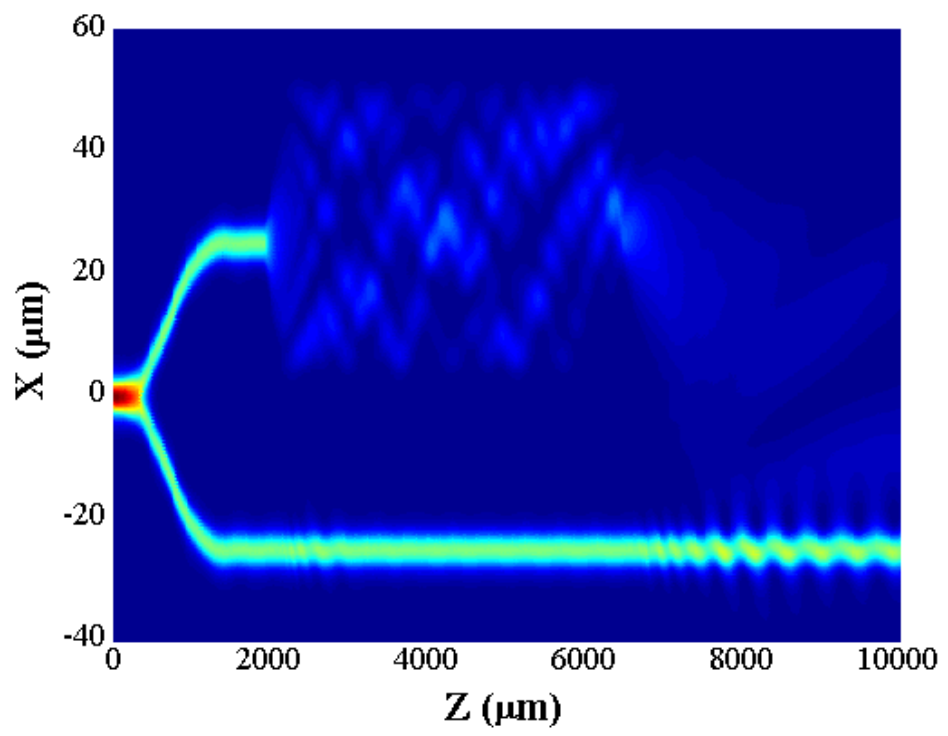

(b)

Fig.3. Modelled results for light propagation within the proposed configuration at two wavelengths (a) $1500 \mathrm{~nm}$ and (b) $1600 \mathrm{~nm}$. 
To investigate the fabrication tolerance of the designed structure, we calculate the spectral response of the MMI structure with a disturbance to the optimised parameters. Corresponding results are presented in Fig.4. A disturbance of $\pm 5 \mu \mathrm{m}$ to the length of the MMI section leads to a maximal variation in transmission of $0.43 \mathrm{~dB}$. For a manufacturing tolerance range of $\pm 0.2 \mu \mathrm{m}$ to the width of the MMI section, the position of the input waveguide $\mathrm{x} 1$, and the position of the output waveguide $x 2$, it is $4.0 \mathrm{~dB}, 0.14 \mathrm{~dB}$, and $0.51 \mathrm{~dB}$ respectively. From these results, we can see that the designed device has a good fabrication tolerance except in regard to the width of the multimode waveguide section. However, from Fig. $4 \mathrm{~b}$ it can be seen that nevertheless the three spectral responses are all suitable for wavelength measurements.
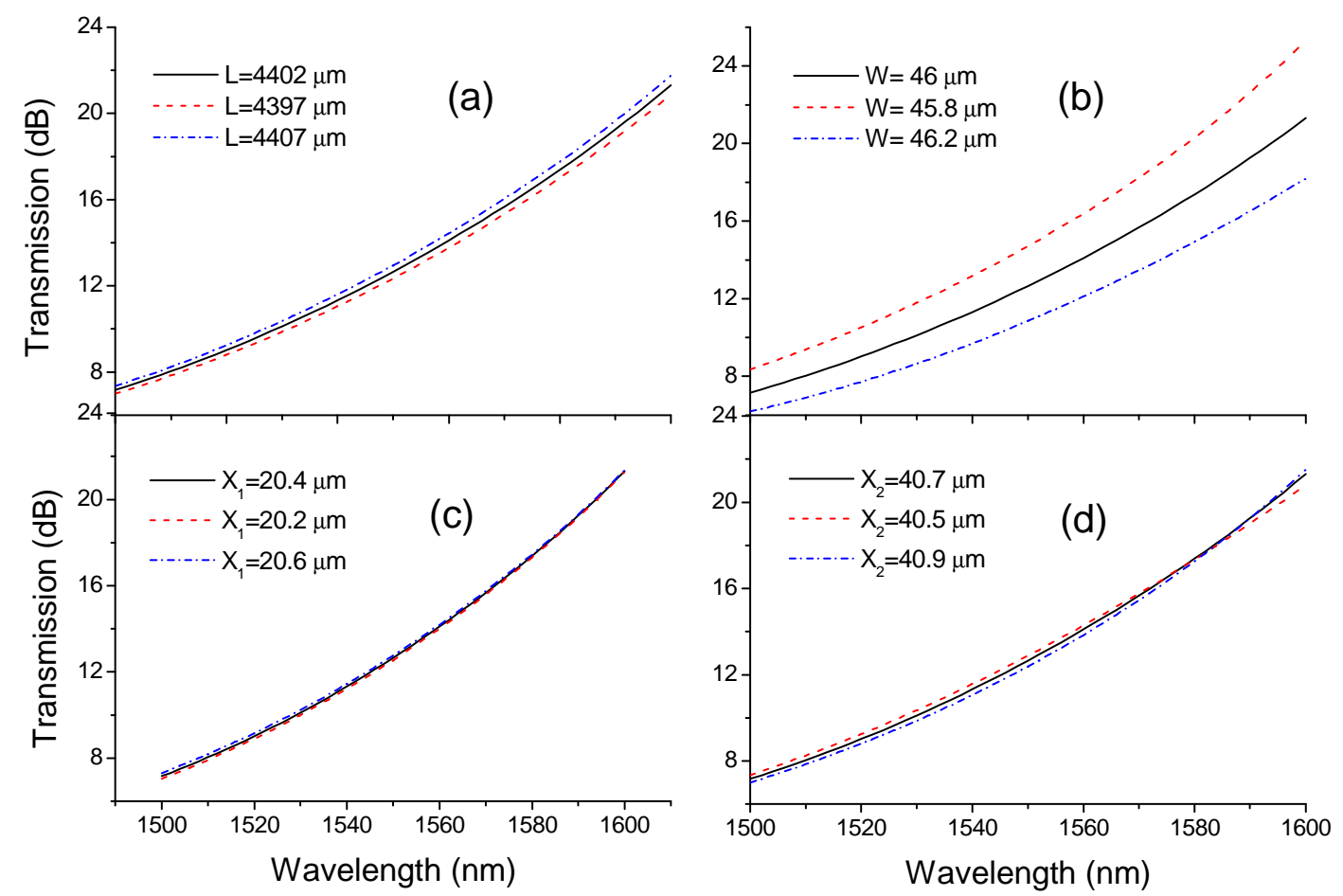

Fig.4. Spectral response of the designed structure with disturbed parameters a) $L=4402 \pm 5 \mu \mathrm{m} ; \mathrm{b}$ ) $\mathrm{w}=46 \pm 0.2 \mu \mathrm{m} ; \mathrm{c}) \mathrm{x} 1=20.4 \pm 0.2 \mu \mathrm{m}$; d) $\mathrm{x} 2=40.7 \pm 0.2 \mu \mathrm{m}$.

\section{CONCLUSION}

An integrated ratiometric wavelength monitor has been presented, which uses the multimode interference structure as an edge filter. For the MMI structure, the length and width of the multimode waveguide section, and positions of the input and output waveguides are optimised using the global optimisation algorithm-simulated annealing. Based on the simulated annealing optimization algorithm, the final calculated results for the multimode waveguide section are $\mathrm{W}=46 \mu \mathrm{m}, \mathrm{L}=4402 \mu \mathrm{m}, \mathrm{x}_{1}=20.4 \mu \mathrm{m}$, and $\mathrm{x}_{2}=40.7 \mu \mathrm{m}$. A specific spectral response has been achieved with the MMI based edge filter: 1) the baseline loss is $7.17 \mathrm{~dB} @ 1500 \mathrm{~nm}$ and 2) the discrimination range (the power difference between transmission at $1600 \mathrm{~nm}$ and $1500 \mathrm{~nm}$ ) is $14.13 \mathrm{~dB}$. These values for baseline loss and discrimination range are typical of those found in real systems and are competitive with other approaches to edge filter implementation. The designed MMI structure has a spectral response suitable for wavelength measurement and it also has a good fabrication tolerance shown by the disturbance analysis of the optimised parameters. 


\section{ACKNOWLEDGEMENT}

P. Wang is funded by the Irish Research Council for Science, Engineering and Technology, co-funded by the Marie-Curie Actions under FP7. G. Brambilla gratefully acknowledges the Royal Society (London, UK) for his University Research Fellowship. Q. Wu gratefully acknowledges the support of Science Foundation Ireland under grant no. 07/SK/I1200.

\section{REFERENCES}

[1] Lepley, J. J. and Siddiqui, A. S., "Primary referenced DWDM frequency comb generator," IEE Proc. Optoelectron. 146(3), 121-124 (1999).

[2] Sookdhis, C., Mei, T. and Djie, H. S., "wavelength monitoring with low-constrast multimode interference waveguide," IEEE Photonic. Technol. Lett. 17(4), 822-824 (2005).

[3] Li, H., Zhong, S., Yang, X., Chen, Y. J. and Stone, D., "Full coverage multichannel wavelength monitoring circuit using center-offset phased-array waveguide grating," Electron. Lett. 34(22), 2149-2150 (1998).

[4] Wang, Q., Farrell, G., Wang, P., Rajan G. and Freir, T., "Design of integrated wavelength monitor based on a Y-branch with an S-bend waveguide," Sensors \& Actuators: A. Physical 134(2), 405-409 (2007).

[5] Soldano, L. B. and Pennings, E. C., "Optical multi-mode interference devices based on self-imaging: Principles and applications," J. Lightw. Technol. 13(4), 615-627 (1995).

[6] Sookdhis, C., Mei, T., Djie, H. S. and Arokiaraj, J., "Passive wavelength monitor based on multimode interference waveguide," Opt. Eng. 42(12), 3421-3422 (2003).

[7] Ingber, L. and Rosen, B. "Genetic algorithms and very fast simulated reannelaing: a comparison," Math. \& Comp. Model. 16, 87-100 (1992).

[8] Boudet, T., Chaton, P., Herault, L., Gonon, G., Jouanet L. and Keller, P., "Thin-film designs by simulated annealing," App. Opt. 35(31), 6219-6226 (1996).

[9] El-Agmy, R., Bulte, H., Greenaway A. H. and Reid, D. T., "Adaptive beam profile control using a simulated annealing algorithm," Opt. Express 13(16), 6085-6091 (2005).

[10] Wang, Q., Farrell G. and Freir T., "Optimal design of broadband linear polarization converters/switches,” Journal of Optics A: Pure Appl. Opt 7(8), 47-50 (2005).

[11] Kawano, K. and Kitoh, T., "Introduction to optical waveguide analysis, Solving Maxwell's equations and the Schrödinger equation,” John Wiley \& Sons, Inc. 5, 165-233 (2001). 